\title{
Success Rate of Dental Implants Placed in the Atrophic Posterior Maxilla with Intentional Sinus Floor Perforation in Lieu of Indirect Sinus Augmentation: A Retrospective Report of 26 Consecutive Patients and Literature Review
}

\author{
Khurram M. Shahzad1, Andrew Q. Madson², Evan M. Shipp³, Andrew W. Ellis ${ }^{4}$ \\ ${ }^{1}$ Oral and Maxillofacial Surgeon, US Air Force Residency Program, David Grant Medical Center, Travis Air Force Base, Fairfield, \\ CA, USA \\ ${ }^{2}$ Oral and Maxillofacial Surgeon, US Air Force Hospital, Lakenheath, UK \\ ${ }^{3}$ Oral and Maxillofacial Surgery Residency Program, San Antonio Military Medical Center (SAMMC), San Antonio, TX, USA \\ ${ }^{4}$ US Air Force Hospital, Lakenheath, UK \\ Email:kshahzad7@gmail.com, khurram.shahzad.1@us.af.mil
}

How to cite this paper: Shahzad, K.M., Madson, A.Q., Shipp, E.M. and Ellis, A.W. (2017) Success Rate of Dental Implants Placed in the Atrophic Posterior Maxilla with Intentional Sinus Floor Perforation in Lieu of Indirect Sinus Augmentation: A Retrospective Report of 26 Consecutive Patients and Literature Review. Open Journal of Stomatology, 7, 113-120. https://doi.org/10.4236/ojst.2017.72007

Received: July 14, 2016

Accepted: February 21, 2017

Published: February 24, 2017

Copyright (๑) 2017 by authors and Scientific Research Publishing Inc. This work is licensed under the Creative Commons Attribution International License (CC BY 4.0).

http://creativecommons.org/licenses/by/4.0/ (c) (i) Open Access

\begin{abstract}
Purpose: To assess surgical success rate of placing dental implants in the atrophic posterior maxilla engaging the maxillary sinus floor in patients with inadequate posterior maxillary alveolar bone height (PMABH). Methods: 26 patients with PMABH of $7-10 \mathrm{~mm}$ had 39 implants placed between July 2012 and June 2014. These implants were placed protruding apically $1-3 \mathrm{~mm}$ into the maxillary sinus engaging the sinus floor. At stage one, implants and cover screws were placed, followed by stage two, 5 - 6 months later. The implants were considered osseo-integrated in the absence of symptoms, mobility and inflammation. Periapical radiographs were taken to rule out peri-implant radiolucency. Restorative treatment was completed 2 months thereafter. Patients were scheduled to have a dental exam (6 - 12 months after delivery of prosthesis) and the implants were evaluated clinically and radiographically. A retrospective review was conducted to assess the surgical success rate of this treatment modality. Clinic charts, radiographs and digital implant log were reviewed for all patients with PMABH of $7-10 \mathrm{~mm}$ who received dental implants in the posterior maxilla to determine the success rate of this treatment modality. Results: 26 patients who had 39 implants placed were included in the study. All implants (100\%) were osseo-integrated at the time of uncovering (stage 2). None of the implants ( $0 \%$ ) had mobility or inflammation around them. Stage 2 was successfully performed on all 39 implants (100\%). All implants $(100 \%)$ in these patients had been restored, and were in good condition
\end{abstract}


without mobility or inflammation, and had been functioning well 6 - 12 months after restoration. Conclusion: This study suggests that a high success rate can be attained placing dental implants in the posterior maxilla while perforating and engaging the cortical maxillary sinus floor. A future prospective study that compares this technique with the internal sinus lift would likely help elucidate this suggestion.

\section{Keywords}

Posterior Maxilla, Implants, Sinus Perforation

\section{Introduction}

Dental implant placement has become a popular and successful procedure for replacing missing teeth. Adequate posterior maxillary alveolar bone height (PMABH) and width are necessary for optimal outcomes. Bone height deficiency can be especially problematic in the posterior maxilla due to sinus pneumatization after extraction of molar and often premolar teeth. Posterior maxillary alveolar height can be increased successfully via a lateral wall sinus augmentation also known as a "direct sinus lift". Generally, when this bone deficiency is greater than $3 \mathrm{~mm}$, a "direct sinus lift" is performed through the lateral maxillary sinus wall by lifting the sinus membrane under direct visualization and placement of bone graft with or without concomitant implant placement. For example, if a $10 \mathrm{~mm}$ long implant is planned but less than $7 \mathrm{~mm}$ of bone height is available, then the direct sinus lift is performed. If however, the posterior maxillary bone deficiency is minimal ( $1-3 \mathrm{~mm})$, it is often managed using the "indirect sinus lift" [1]. For example, if a $10 \mathrm{~mm}$ long implant is planned and $7 \mathrm{~mm}$ - $9 \mathrm{~mm}$ of bone is available, then an "indirect sinus lift" is performed. An implant osteotomy is made short of the maxillary sinus floor by about $2 \mathrm{~mm}$ and osteotomes are used to fracture up the sinus floor and particulate bone graft material is placed between the created implant osteotomy and the fractured sinus floor, resulting in increased eventual PMABH. This technique can be utilized without a bone graft as well [2]. Usually an implant is placed at the same time. With bone deficiency of $1-3 \mathrm{~mm}$, other options include use of short implants [3] (less than $7 \mathrm{~mm}$ ) or placing the implant without the internal sinus lift [4]. In the case of the latter, a conventional implant osteotomy is made perforating the sinus floor cortex and the implant is placed engaging the sinus floor. The apical $1-3 \mathrm{~mm}$ of the implant is through the sinus floor cortex and into the maxillary sinus. Several studies and case reports have shown that implants placed in this manner show excellent success rates [4] [5]. At our treatment facility, if the posterior maxillary height deficiency is greater than $3 \mathrm{~mm}$, a direct sinus lift is performed with a particulate bone graft to increase bone height. If the deficiency is $1-3 \mathrm{~mm}$, an implant osteotomy is made with sinus perforation and the implant is placed and the internal sinus lift is not performed. This study was conducted to assess the success rate of this procedure in our patient population. 


\section{Methods}

The clinic charts as well as the digital implant log were reviewed for all patients who received dental implant treatment of the posterior maxilla from July 2012 -June 2014 at the department of Oral and Maxillofacial Surgery, U.S. Air Force base, RAF Lakenheath, UK. This study was approved by the U.S. Air force Research Oversight \& Compliance Division. Patients with symptoms of sinus disease or radiographic signs of maxillary sinus pathology were excluded. Patients who had more than $10 \mathrm{~mm}$ of PMABH were excluded as well as patients who had less than $7 \mathrm{~mm}$ of bone height. Other exclusion criteria included uncontrolled or poorly diabetes mellitus and smoking. The study variables were patient gender, implant width ( $4 \mathrm{~mm}$ or $5 \mathrm{~mm}$ ) and location (premolar or molar). 26 patients had PMABH of $7-10 \mathrm{~mm}$ and had 39 implants placed in the posterior maxilla by the author (KMS). Patients ranged in age from 20 to 52 years with a mean age of 31 years. 14 patients were male and 12 were female. All implants were placed 1, 2 or $3 \mathrm{~mm}$ into the maxillary sinus. All implants were $10 \mathrm{~mm}$ long. 7 implants ( $4 \mathrm{~mm}$ wide) were placed in the premolar position. 32 implants ( $5 \mathrm{~mm}$ wide) were placed in the molar position. Surgical phase of the treatment was staged. Implants and cover screws were placed during Stage one surgery. Implant osteotomy was made using a $2 \mathrm{~mm}$ twist drill to the length of the planned implant perforating the maxillary sinus bony floor and membrane. The osteotomy was then sequentially widened at the same length and the appropriate width implant (3i, Biomet, Palm Beach Gardens, Florida) was placed at a torque value of $>35 \mathrm{Ncm}$. All patients were prescribed Augmentin $875 \mathrm{mg}$ (GlaxoSmithKline, Brentford, UK) BID for 5 days. No patients in the study had a Penicillin allergy. Stage two (replacement of the cover screw with healing abutment) was performed 5 - 6 months after by the same surgeon (KMS). At Stage two the implants were considered osseo-integrated (surgically successful) in the absence of symptoms, mobility, erythema, tenderness or purulence. This was complemented by periapical radiographs to confirm the absence of peri-implant radiolucency. Patients were then treated at the prosthodontic department for prosthesis fabrication and treatment was completed by 2 months after stage two. Patients were then scheduled to have a routine dental exam (6 months to a year after delivery of prosthesis) at which point the implants were evaluated clinically and radiographically. Symptoms and signs were recorded pertaining to the implants. Recorded symptoms were subjective report of pain and/or swelling in the area(s) of implant placement. Recorded signs were erythema, tenderness, purulent drainage, presence of granulation tissue and implant mobility at time of stage 2. If patients had moved, they were contacted via telephone and questioned about symptoms. Their digital radiographs were reviewed to rule out any radiolucency between implants and bone.

\section{Results}

26 patients who had 39 implants placed were included in the study. All implants $(100 \%)$ were osseo-integrated at the time of uncovering (stage 2$)$. None $(0 \%)$ of 
the patients reported symptoms of pain or swelling since one week after surgery. None of the implants (0\%) had mobility, inflammation (erythema, tenderness or drainage) or granulation tissue around them. No (0\%) radiographs showed peri-implant radiolucency. Stage 2 was successfully performed on all implants (100\%). 24 patients had prostheses fabricated (for 37 implants) at our facility by the prosthodontic department. 2 patients moved out of the area and received implant prostheses at a different facility. Of the 24 who received implant prostheses at our facility, 21 (88\%) were seen between 6 months to one year for a dental exam. All implants (100\%) in these patients were in good condition without mobility or inflammation and had been functioning well. 3 patients (12\%) had moved out of the area and could not be examined clinically. These 3 patients were contacted by phone and none reported symptoms. They also denied any unscheduled dental visits with regards to the implants. The 2 patients who moved out of the area (and received one implant prosthesis, each at a different military facility) were also contacted by phone and denied any symptoms or unscheduled dental visits with regards to the implants. Their radiographs showed restored implants without any peri-implant radiolucencies as well.

\section{Discussion}

Deficient posterior maxillary alveolar bone height can complicate surgical placement of dental implants. When the PMABH deficiency is $1-3 \mathrm{~mm}$, the common treatment modalities utilized are the indirect sinus lift or the use of short implants. Due to institutional restrictions on implant brand, dental implants that are shorter than $8 \mathrm{~mm}$ are not used at our facility. Because of this, the options available were to perform a direct sinus lift, an indirect sinus lift or place implants without a sinus lift where the apical $1-3 \mathrm{~mm}$ of the implant protrudes into the maxillary sinus. We utilized the last option where the maxillary sinus floor was perforated to engage the cortical bone to place the implant (Figure $1 \&$ Figure 2). We observed a success rate of $100 \%$ in our patient population. Nooh et al. reported on a series of 56 patients who received 63 implants [4], where only

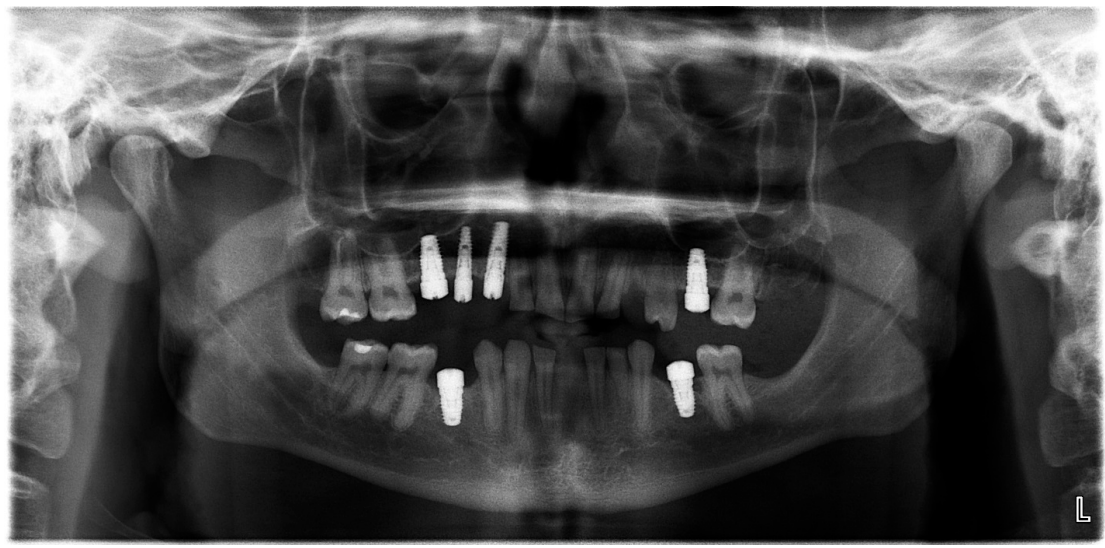

Figure 1. Postoperative (stage 2) Panoramic radiograph of Left maxillary first molar implant placed with sinus floor perforation and $2 \mathrm{~mm}$ of apical implant protruding into Left maxillary sinus. 


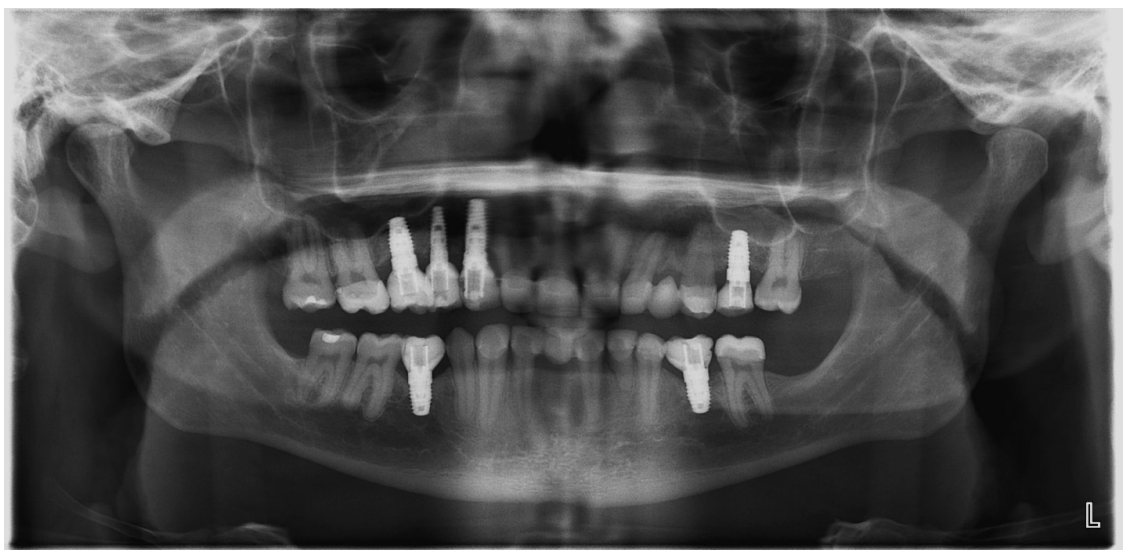

Figure 2. Panoramic radiograph of the same patient in Figure 1 nine months after prosthetic restoration.

one of 63 implants failed and had to be removed. He reported a $98.4 \%$ success rate with only one implant failure after a follow-up period of one year. In our study, we observed a high success rate in general but also comparable to other modalities of direct sinus lift, the indirect sinus lift and the use short dental implants [1] [2] [3] which show success rates higher than $90 \%$. That may simply be due to the relatively small sample size and short term follow-up. Increasing the sample size and longer follow-up would surely improve the power of the study. Some other limitations of this study are that it is retrospective and lacks a control group and that some of the patients were lost to follow up and were not clinically evaluated by the authors. In the case of the latter, it was assumed that the implants were successful in the absence of subjective or objective findings based on a phone interview and review of chart entries. In the future, a prospective study that compares the three different treatment modalities would be beneficial to answer the question which modality has the best success rate for dental implants placed into the posterior maxilla with alveolar bone height deficiency of $1-3 \mathrm{~mm}$.

There are numerous benefits to this technique. One benefit is that it is more efficient. It does not require extra time to perform a direct or indirect sinus lift. Also no bone grafting is done which makes for a more cost-effective procedure. Patient comfort may be a factor when comparing to an indirect sinus lift where an osteotome and mallet are used to fracture up the sinus floor. Some patients especially those not sedated for the procedure might find the tapping of the mallet onto the osteotome unpleasant. Not performing an indirect sinus lift would also make for a quicker procedure since it is one less step to perform. We also think that engaging the cortical bone of the sinus floor during implant placement helps to maximize the initial stability of the implant. Takahiro et al. [6] showed that new bone formation takes place in non-grafted maxillary sinuses as long as space is maintained. It is possible that the $1-3 \mathrm{~mm}$ of the apical implant surface protruding into the maxillary sinus can have bone growth adjacent to it, increasing the surface area of the implant that is covered by bone (Figure 3 and Figure 4). 


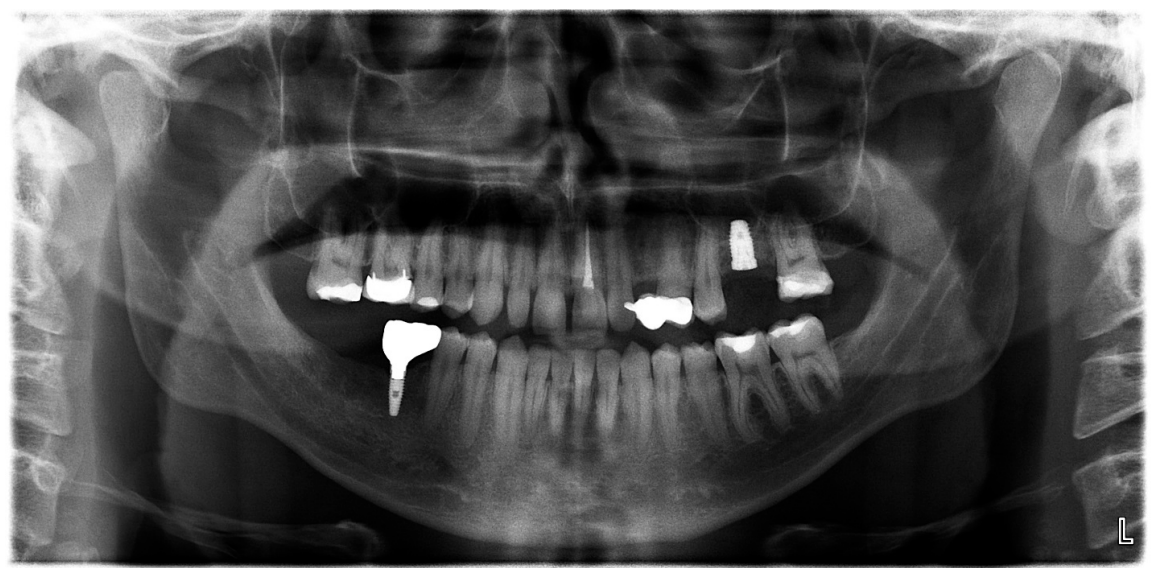

Figure 3. Postoperative (stage 1) Panoramic radiograph of another patient in whom the Left maxillary first molar implant was placed with sinus floor perforation and $2 \mathrm{~mm}$ of apical implant protruding into Left maxillary sinus.

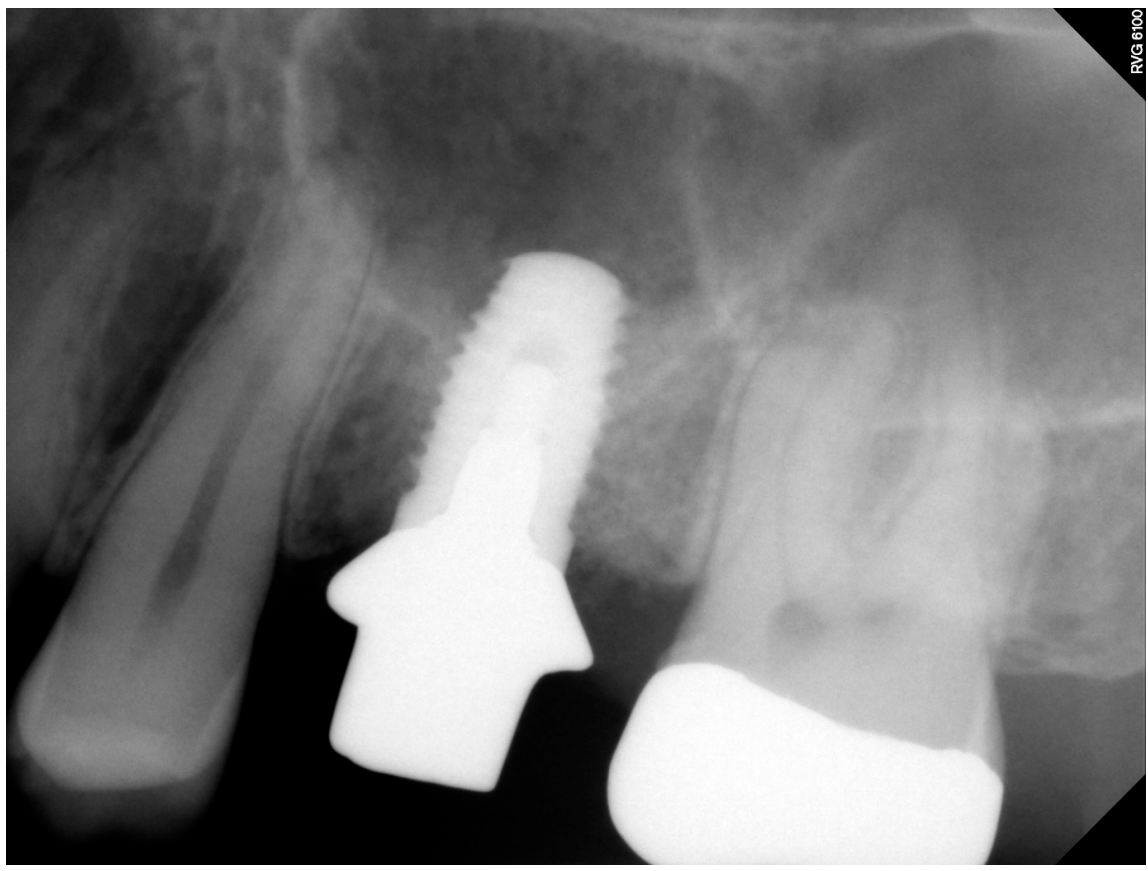

Figure 4. Periapical radiograph of same patient at time of stage 2 (6 months after stage 1) with radiopacities near implant apex suggesting bone growth.

Naturally clinicians may have some hesitation in perforating the sinus floor to place a foreign body that protrudes into the sinus. In theory, this can lead to sinus problems like sinusitis and its sequelae. Nooh pointed out that the maxillary sinus membrane is torn very often during Lefort I osteotomy and is usually of no negative consequence [4]. Barone et al. reported that of 124 sinus augmentations performed in their group, $25 \%$ were associated with a sinus membrane perforation [7]. They concluded that the sinus membrane perforation was not shown to be a significant factor in the rate of implant complications. Numerous case reports of accidental sinus perforation during implant placement conclude that the perforation did not lead to any serious sequela or morbidity [8] [9]. 
An example of a prosthesis often placed trans-antrally is the Zygomatic implant in patients with severe posterior maxillary alveolar bone deficiency. Even though these implants often pass through the maxillary sinus before engaging the body of the zygoma, they are rarely associated with significant sinus problems after placement. In their retrospective cohort study, D'Agostino et al. [10] concluded that placement of zygomatic implants does not seem to be associated with severe rhinosinusitis complications. This underscores the point that immobilized foreign bodies that protrude into the maxillary sinus are not likely to cause significant sinus problems as long as there is no oral-antral communication. Similar to the direct or indirect sinus lift procedure, we would not recommend this technique in patients with acute or chronic maxillary sinusitis. Our study suggests that placing implants $1-3 \mathrm{~mm}$ into the maxillary sinus to engage the cortex is a safe modality with a good success rate. It may offer a success rate similar to placing them with an internal sinus lift procedure (with or without bone grafting). It may also be initially more stable, more efficient, more comfortable and more cost effective than the indirect sinus lift. A future controlled study that prospectively compares these modalities would likely elucidate this suggestion.

The views expressed in this article are those of the authors and do not reflect the official Policy of the U.S. Air Force, the Department of Defense or other departments of the United States government.

\section{References}

[1] Halpern, K., Halpern, E. and Ruggiero, S. (2006) Minimally Invasive Implant and Sinus Lift Surgery with Immediate Loading. Journal of Oral and Maxillofacial Surgery, 64, 1635-1638. https://doi.org/10.1016/j.joms.2006.04.043

[2] Perez-Martinez, S., Martorell-Calatayud, L., Penarrocha-Oltra, D., Garcia-Mira, B. and Penarrocha-Diego, M. (2015) Indirect Sinus Lift without Bone Graft Material: Systemic Review and Meta-Analysis. Journal of Clinical and Experimental Dentistry, 7, e316-e331. https://doi.org/10.4317/jced.51716

[3] Urdaneta, R.A., Haher, S., Leary, J., Emanuel, K.M. and Chuang, S.K. (2012) The Survival of Ultra Short Locking-Taper Implants. The International Journal of Oral \& Maxillofacial Implants, 27, 644-654.

[4] Nooh, N. (2013) Effect of Schneiderian Membrane Perforation on Posterior Maxillary Implant Survival. Journal of International Oral Health, 5, 28-34.

[5] Branemark, P.I., Adell, R., Albrektsson, T., Lekholm, U., Lindstrom, J. and Rockler, B. (1984) An Experimental and Clinical Study of Osseointegrated Implants Penetrating the Nasal Cavity and Maxillary Sinus. Journal of Oral and Maxillofacial Surgery, 42, 497-505. https://doi.org/10.1016/0278-2391(84)90008-9

[6] Takahiro, K., Issei, M., Norie, H. and Tetsuo, S. (2012) New Bone Formation in Non Grafted Sinus Lifting with Space-Maintaining Management: A Novel Technique Using a Titanium Bone Fixation Device. Journal of Oral and Maxillofacial Surgery, 70, e217-e224. https://doi.org/10.1016/j.joms.2011.10.025

[7] Barone, A., Santini, S., Sbordone, L., Crespi, R. and Covani, U. (2006) A Clinical Study of the Outcomes and Complications Associated with Maxillary Sinus Augmentation. The International Journal of Oral \& Maxillofacial Implants, 21, 81-85. 
[8] Hernandez-Alfaro, F., Torradeflot, M.M. and Marti, C. (2007) Prevalence and Management of Schneiderian Membrane Perforations during Sinus-Lift Procedures. Clinical Oral Implants Research, 19, 91-98.

[9] Becker, S.T., Terheyden, H., Steinriede, A., Behrens, E., Springer, I. and Wiltfang, J. (2008) Prospective Observation of 41 Perforations of the Schneiderian Membrane during Sinus Floor Elevation. Clinical Oral Implants Research, 19, 1285-1289. https://doi.org/10.1111/j.1600-0501.2008.01612.x

[10] D’Agostino, A., Trevisiol, L., Favero, V., Pessina, M., Procacci, P. and Nocinic, P.F. (2016) Are Zygomatic Implants Associated with Maxillary Sinusitis? Journal of Oral and Maxillofacial Surgery, 74, 1562-1573.

https://doi.org/10.1016/j.joms.2016.03.014

Submit or recommend next manuscript to SCIRP and we will provide best service for you:

Accepting pre-submission inquiries through Email, Facebook, LinkedIn, Twitter, etc. A wide selection of journals (inclusive of 9 subjects, more than 200 journals)

Providing 24-hour high-quality service

User-friendly online submission system

Fair and swift peer-review system

Efficient typesetting and proofreading procedure

Display of the result of downloads and visits, as well as the number of cited articles Maximum dissemination of your research work

Submit your manuscript at: http://papersubmission.scirp.org/

Or contact ojst@scirp.org 